\title{
A review on global prevalence and recent advancements in the genetics of psoriasis
}

\begin{abstract}
Psoriasis Vulgaris is a complex auto immune disorder which results in poorly differentiated hyper proliferative keratinocytes. Psoriasis had been widely studied in the past for a decade using HLA gene and the addition factors which are required for the activation and deactivation of the gene present in the skin lesions of patients suffering with Psoriasis. In this review, we analyze the prevalence and pathology and of Psoriasis across the globe with the recent advancement from candidate gene study HLA genes to Genome Wide Association Study (GWAS) along with the impact of nanotechnology towards the drug delivery of small RNAs of non coding families (ncRNAs) like small interfering RNA (siRNA), miRNA and etc with the help of a carrier molecule to activate and bind to the receptor. In addition to that there were well established studies of miRNA to illustrate its vital role in characterizing the disease pathology of Psoriasis and provide an alternative path towards the treat Psoriasis in future.
\end{abstract}

Keywords: genome wide association approach, hyper proliferative keratinocytes, nanotechnology and disease pathology
Volume 6 Issue 2 - 2017

\section{Daniel Alex Anand, Harishchander}

Anandaram

Department of Bioinformatics, Sathyabama University, India

Correspondence: Department of Bioinformatics, Sathyabama University, Chennai, India, Email Harishchander.a@gmail.com

Received: May 17, 2017 | Published: September 22, 2017

\section{Introduction}

Psoriasis Vulgaris is a chronic inflammatory disease of the skin which affects $2 \%$ of the world population. ${ }^{1,2}$ According to US reports, prevalence of psoriasis is more common in women than men but Indian reports suggest that the prevalence is twice more common in men and the earlier onset of the disease is prior to 15 years. ${ }^{3}$ Psoriasis is prevalent globally. However, statistical studies illustrate its variation among various geographic locations like the occurrence of disease is more common in colder north than in tropics. ${ }^{4,5}$ In larger scale, the population prevalence of psoriasis has been reported in a range between 0 to $11.8 \%{ }^{6,7}$ Since, Psoriasis is a chronic and multifactorial autoimmune disorder in skin, there is an abnormal hyper proliferation in keratinocytes, arised due to the activation of T-cells which produces a rich amount of arachidonic acid which leads to generation of various pro inflammatory mediators like PGs, LTs, cytokines and adhesion molecules via MAPK/AP-1, EARK1/2 and protein kinase-C (PKCs) activation pathways. ${ }^{8}$ In order to treat Psoriasis there is a need for the incorporation of naturally occuring bioactives like, omega $(\omega)-3$ fatty acids (i.e. EPA and DHA) in a dose dependent manner which results in the inhibition of various pro-inflammatory mediators. Metabolization of EPA and DHA leads to the dampening of inflammation and higher resolution of abnormalities in skin. In case of natural treatment the synthesis of $\omega-3$ PUFA-derived lipid mediators, namely resolvins and protectins have been widely used alone or in combination with other drugs in the treatment. Despite of their meritorious visages, the use of these bioactives is associated with several hiccups like higher range of unstability and vulnerable to degradation due to lipid peroxidation. This treatment is also resulted in poor and inconsistent bioavilability by oral and topical administration.

The potential use of nanomedicines in the delivery of such bioactives has gained wider attention Owing to their promise in enhancing the characteristics of bioavailability, improved stability and Better efficacy. Moreover, barriers in the effective delivery of $\omega-3$ fatty acids and how nanomedicines can be fit in the scope of its therapeutic delivery in psoriasis have also been addressed but it still has a long way to go with multidisciplinary aspects like Pharmacogenomics. Despite numerous advantages the application of EPA-DHA as $\omega-3$ fatty acids as a therapeutics in the management of psoriasis is still in an initial stage and the approach of nanomedicines to achieve high bioavailability in delivery with safety and stability of $\omega-3$ fatty acids have shown a promising area for the future in psoriasis management. In order to treat psoriasis with respect to genetic approach, a novel fusogenic nucleic acid lipid particle (F-NALP) system containing two therapeutic nucleic acids namely anti-STAT3 siRNA (siSTAT3) and anti-TNF- $\alpha$ siRNA ( siTNF- $\alpha$ ) were employed along with a novel cationic amphiphilic lipid in oleyl chains were synthesized and used in the nano carrier system and the therapeutic efficacies of F-NALPs were assessed using an imiquimod-induced psoriatic-like plaque model. ${ }^{9}$

In case of treating Psoriasis, a Conventional method of drug delivery systems is inefficient to provide a target effect due to higher bioavailability with its short half life and instability. In such cases a Novel Drug Delivery Systems (NDDS) is required. Certain examples of such systems are liposomes, niosomes, microemulsions, transferosomes, ethosomes, emulsomes, invasomes, dendrimers, nanoparticles, hydrogel, etc. ${ }^{10-12}$ A Novel drug delivery system targets the tissues through skin layers and provides a better therapy for topical treatment of psoriasis. Stratum corneum (SC) is the major challenge for the drug to get into the target tissues via skin layers.

Penetration enhancers are added in the drug carriers for increasing the penetration capacity of drug through the outermost layer of the skin. The most favorable drug delivery should provide high penetration through SC and should not cause any irreversible changes to the skin barrier. ${ }^{13-15}$ Here, the main challenge is to address the transdermal delivery of drugs because there will be a variability in the percutaneous absorption due to site, disease, age and etc. Skin irritation may happen when the toxicities due to drug are more. The first pass metabolic effect of skin is also another challenge for topical 
delivery. ${ }^{16-18}$ Novel drug delivery systems have lot of advantages. They increase safety and efficacy levels.

Drug targeting specificity and lowering systemic drug toxicity are the important merits of DDS. They also they have the ability to improve absorption rates and will prevent biochemical degradation of pharmaceuticals ${ }^{19-21}$ and skin is mostly considered for the root of drug delivery because it is the largest and outermost organ of the human body. Among three important layers of skin, epidermis functions as a protective barrier of the body. ${ }^{22-24}$ There are a lot of blood vessels and layers present in the epidermis. Sub layers are also present in this outermost layer such as stratum lucidum, stratum corneum, stratum spinosum, stratum granulosum, and stratum germinativum. Dermis is present beneath the outermost layer, which is composed of connective tissues. ${ }^{25,26}$ Hypodermis is situated under the dermis layer. For the treatment of psoriasis, percutaneous absorption of drugs is one of the widely accepted ways of drug delivery. The challenge that offered by topical treatment is the presence of SC as a barrier. ${ }^{27-30}$ Conventional forms of drug delivery through skin have come across with many side effects and other application difficulties.

Disruption of SC and targeting to the deeper layers of skin are not possible with ointments, creams etc. So, novel dermal delivery systems help to overcome these limitations, thereby enhance the bioavailability and potential of drug and are widely used for the treatment for psoriasis recently. ${ }^{31}$ Since, Skin is the largest organ of humans which regenerates throughout the entire life of every individual and serves as an outermost barrier for preventing the internal organs from dehydration. ${ }^{32-34}$ It has a system for maintaining the regulatory mechanism of mediators with local or systemic effects. ${ }^{35-37}$ As a common skin disease, psoriasis is chronic, auto immune and a complex genetic disorder which affects around $2 \%$ of world population. Psoriatic skin contains certain symptoms of inflammation which are raised as scaly lesions. ${ }^{38-40}$ There are three types of cellular alterations in psoriatic skin i.e. abnormal differentiation of keratinocyte, hyperproliferation of keratinocyte and infiltration of immune cells. ${ }^{41}$ Studies on the molecular components and cellular pathways of inflammation are one of the major contributions to understand the pathogenesis of psoriasis. ${ }^{42-45}$ The interplay between the genetic and environmental factors influence the onset and progression of psoriasis. ${ }^{46,47}$ Recent studies on miRNAs, illustrate the fact that it is a novel regulator of gene expression and it plays a vital role in psoriasis.

Maturation of miRNAs involves multiple steps and initially two intermediate forms of miRNAs, primary (pri-) and precursor (pre-) miRNAs, are produced sequentially. In this process, RNase III enzyme Drosha and partner double-stranded RNA (dsRNA) binding protein Dgcr8 cleave pri-miRNAs to produce hairpin-shaped premiRNAs that are recognized by Exportin5 and are subsequently transported from nucleus to cytoplasm. There is another RNase III enzyme called Dicer which cleaves the pre-miRNAs to release $\sim 22-$ nt double-stranded RNA duplexes (namely miRNA/miRNA*duplexes) with $\sim 2-$ nt 3' overhangs. One strand of a RNA duplex is termed mature miRNA which is further loaded into an Argonaute protein in the RNA-induced silencing complex (RISC) to exert its regulatory function on the basis of its binding with the target transcripts. ${ }^{48,49}$ Since, there is no permanent cure for Psoriasis in Current stage; we analyze the inflammatory response by the scaly lesions with a widely aberrant gene expression. ${ }^{50}$ Psoriasis was thought to be initiated by the complex interactions between environmental and genetic factors. Skin lesions in Psoriasis are characterized by the hyperproliferation and aberrant differentiation of keratinocytes by the process of in filtering the inflammatory cells into the dermis and epidermis. ${ }^{51}$ Psoriasis skin lesions are characterized by hyperproliferation and aberrant differentiation of keratinocytes and infiltration of inflammatory cells into the dermis and epidermis. Recent studies on the increase or decrease in the gene expression levels of patients with psoriasis, revealed the fact that miRNAs play critical roles in regulating as a class of post transcriptional genes in Psoriasis. ${ }^{52}$ Micro RNA is a family of non coding RNA (ncRNA) which was discovered in 1993, it consist of 19-25 nucleotides and regulates the expression of approximately $30 \%$ of protein-coding miRNAs in humans.

Base pairing at the position 2-8 nucleotides enrich are relative to the $5^{\prime}$ end of the small RNA is termed as the "seed" region and it appears to be important for target recognition. ${ }^{53} \mathrm{~A}$ unique miRNA can regulate the expression of hundreds of proteins and the expression of a specific protein may be controlled by several miRNAs. ${ }^{54}$ The sequence conservation of most miRNAs lies between the distantly related organisms to suggest the impact of a strong evolutionary pressure ${ }^{55}$ and they have been shown to participate in many fundamental life processes like development, differentiation, organogenesis, growth control and apoptosis. Accordingly, deregulation of miRNA expression has been shown to contribute to cancer, heart diseases, infectious diseases, inflammatory diseases, and other medical conditions, making them potential targets for medical diagnosis and therapy. ${ }^{56}$ Initially, Lee et al., ${ }^{22}$ had found lin-4 as a regulator of developmental timing in nematode Caenorhabditis elegans. ${ }^{57}$ After severalyears, Reinhart et al. ${ }^{36}$ had discovered lethal-7 (let-7) gene in Caenorhabditis elegans. ${ }^{58}$ At present, 2500 miRNAs are in the human genome. Majority of miRNA are intragenic. ${ }^{59}$ Micro RNAs are initially transcribed as part of an RNA stem-loop that in turn forms part of a several hundred nucleotides long miRNA precursor miRNA (pri-miRNA) ${ }^{60}$

Mature miRNA is a part of an RNA-induced silencing complex (RISC) which contains Dicer and many associated proteins. ${ }^{61}$ Since miRNA is involved in the functioning of eukaryotic cells, dysregulation of miRNA been associated with disease and a miR2Disease database contain documents with known relationships between miRNA dysregulation and human disease. ${ }^{61}$ Micro RNAs can bind to target messenger RNA (mRNA) transcripts of protein-coding genes and negatively control their translation or cause mRNA degradation and the key factor is to identify the importance miRNA target with accuracy. A detailed review for the advances in the miRNA target identification methods and available resources has been published by Zheng et al. ${ }^{61}$

Next-Generation sequencing (NGS) is a sequencing technology with the incorporation of high-throughput methods which has the capability of profiling the expression of various RNA in different species with a resolution of single-nucleotide on the basis of genomewide scaling. ${ }^{62,63}$ NGS sequences RNA transcripts directly and hence it is able to facilitate the de novo discovery of genes with novel miRNA. ${ }^{63}$ Recent studies on NGS had discovered a pool of miRNAs and miRNA-like RNAs on the basis of their variants. These diverse miRNAs include canonical and noncanonical miRNAs,${ }^{64}$ miRNA-like RNAs, and miRNA isoforms. ${ }^{64}$ Canonical miRNAs are generated from a biogenesis pathway that requires Drosha and Dicer. Noncanonical miRNAs are produced from alternative pathways in biogenesis where Drosha is not involved. The first example of noncanonical miRNA is the class of mirtrons, which arise from a short nucleotide of sequence length ranges from 60 to 100 bases. Dicer dependent but Dgcr8 independent miRNA-like RNAs can also arise from the formation of local hairpin within the region of larger noncoding RNA (ncRNA). Micro RNAs are typically defined as the most abundant small RNAs 
on pre-miRNA hairpins. Nevertheless, other less abundant but cognate small RNAs from the same pre-miRNAs, which differ by a few bases from miRNAs, have also been reported ${ }^{65}$ and the variants of these miRNA have been named as isomiRs. IsomiRs can function as regular miRNAs ${ }^{65}$ and they often share the mRNA target which is common with their companion miRNAs but in some cases they may also have their own exclusive genes as target. ${ }^{65}$ The emergence of such diverse miRNAs as additional regulators of gene expression with potential functions is complementary to that of canonical miRNAs which reflects the robustness and plasticity of miRNA-mediated regulation of gene expression.

There is an estimate that miRNAs regulate over the one-third portion of protein-encoding mRNAs in humans. ${ }^{66}$ Recent studies on the development of mammalian skin have revealed the fact that that "Interaction between miRNAs and their target mRNAs is vital for the regulating signaling pathways during cell differentiation". ${ }^{66}$ There were also reports on Noncanonical miRNAs, miRNA-like RNAs and isomiRs in normal and psoriatic skin of human. ${ }^{66}$ Dysregulation of miRNAs and their regulated targets has been implicated in the pathogenesis of psoriasis ${ }^{67}$ as well as other forms of disorder of the skin, including malignant melanoma. ${ }^{67}$ The aberrant expression of small non-coding RNAs in psoriatic skin has suggested functional roles of sncRNAs in psoriasis. This emerging theme on miRNAs suggests that these miRNAs have the potency to become a therapeutic target for treating psoriasis

\section{Prevalence of psoriasis in Europe}

A handful of population-based studies were clinically evaluated and it has been reported that the prevalence of disease is highest among Northern Europeans and the landmark of population-based study on the prevalence of psoriasis in a community was conducted by Gunnar Lomholt at the Faroe Islands, an island group situated between Norwegian Sea and North Atlantic Ocean, approximately halfway between Scotland and Iceland [68]. He examined 10,984 inhabitants in the relatively isolated islands and he reported that the prevalence of psoriasis is $2.8 \%$ but a high prevalence has been reported in a population-based study from Norway consisting of 14,667 adult men and women for coronary risk factors and he reported that the overall prevalence of psoriasis was $4.8 \%{ }^{68} \mathrm{~A}$ similar prevalence of $4.2 \%$ were reported in a population-based health survey of 8045 Norwegian twins aged 19-31years based on self-reported psoriasis on a questionnaire. ${ }^{69,70}$

Contradicting the previous reports, population based in prevalence United Kingdom was estimated to be in the range between 1.5 to $2.8 \%{ }^{71,72}$ Another studies with a similar design in semi-urban population of 5395 subjects in east Leicestershire revealed prevalence of $1.48 \%{ }^{73}$ Other two surveys from Germany were examined the adults patients in Augsburg (Southern Germany) with similar prevalence rate like UK but a study in Italy with stratified random sampling and a computer-assisted interview by trained interviewers of about 3660 subjects (age: 45years), gave 3.1\% prevalence weight according to distribution of Italian population. ${ }^{73}$ In France, a self-reported questionnaire-based survey of population revealed $3.58 \%$ prevalence for the spread of Psoriasis from other cutaneous inflammation in skin. ${ }^{73}$

After analyzing the estimated prevalence of clinic-based European and British populations, the results were $6.5 \%$ in Germany, $5.5 \%$ in Northern Ireland, $4.8 \%$ in the west of Scotland and 3.7\% in Barcelona and Spain. ${ }^{73}$ Thus, in Europe the prevalence of psoriasis varies anywhere from 0.6 to $6.5 \%$ and a recent study based on patient report of physician diagnosed psoriasis commissioned by the National Psoriasis Foundation showed prevalence of $1.3 \%$ in AfricanAmericans, which was significantly lower than that in Caucasians $(2.5 \%)$ but in Newfoundland (Canada), the prevalence was estimated to be $2.3 \%{ }^{74}$ Thus, estimates from North America reflect that the dis3ease is obtained from Europe and it reflect the European ethnic origins of most North Americans and African-Americans have a lower prevalence of psoriasis compared to Whites. ${ }^{74}$ Most AfricanAmericans trace their origins from West Africa where the prevalence of psoriasis is lower than elsewhere in Africa. ${ }^{74}$

\section{Prevalence of psoriasis in Africa}

The prevalence of psoriasis in patients attending skin clinics in West Africa is reported to be $0.08-0.9 \%$ in Nigeria, $0.4 \%$ in Ghana, $0.05 \%$ in Mali and $0.3 \%$ in Angola. ${ }^{74}$ However, in East Africa as well as in ethnically mixed populations of Egypt and South Africa the reported prevalence is at higher rate i.e. in Kenya, Uganda and Tanzania the prevalence was reported to be $3.5 \%, 2.8 \%$ and $3 \%$ respectively. ${ }^{75}$ In Egypt, the prevalence was reported to be $3 \%$ and among South African population the Bantu have a lower prevalence compared to Whites, i.e. $1.5 \%$ and $4 \%$ respectively. ${ }^{75}$ Psoriasis was more common in persons of East Indian descent compared to those of African origin. ${ }^{75}$ Similar to data from South America, no psoriasis was found among 'full-blood'. A study consist of Australian Aborigines examined 3000 subjects in central, northern, and southern Australia and it has been found that the prevalence rate is higher in White Australians i.e. a prevalence of $2.57 \%$ was reported among 25,296 patients seen in a private specialist practice in Melbourne and similarly $2.3 \%$ was reported among 1037 subjects after clinically evaluation in a population survey and No psoriasis was seen in a population-based survey of 12,569 subjects in American Samoa in the south Pacific region. ${ }^{75}$

\section{Vital incidences of psoriasis in USA}

There are a fewer studies on the incidence of psoriasis in the general population of USA based Rochesters Epidemiology Project in 1980.Subsequently, from the same project Shbeeb screened all psoriasis cases between 1982 and 1991 and reported an incidence of 107.7 per $100,000 .^{75}$ This increasing trend in Rochester, Minnesota was confirmed in a recent study by Icen et al. ${ }^{50}$ because it suggest an overall age and sex-adjusted annual incidence of psoriasis i.e. 78.9 per 100,000.Incidence of psoriasis increased significantly over time from 50.8 in the period of $1970-1974$ to reach 100.5 per 100,000 in $1995-1999 .{ }^{75}$

\section{Vital incidences of psoriasis in UK}

In the United Kingdom, Huerta et al., ${ }^{49}$ reported a higher estimated incidence of psoriasis during 1996-1997 i.e. 140 per 100,000 patients. ${ }^{75}$ Thus, by reviewing a large number of studies of varying designs and methodological quality on the prevalence of psoriasis conducted over the last 50years, we can make the following conclusions like psoriasis is prevalent worldwide except in certain indigenous populations and on average, the prevalence is higher in those with European ethnicity, especially in the northern part. ${ }^{75}$

\section{Similarities among the global prevalence of psoriasis}

The prevalence of Psoriasis is probably similar in East and North Africa but lower in West Africa. The prevalence is distinctly lower in East Asia and clinic prevalence estimates are consistently higher 
than population-based estimates. The variation between clinical and population study is based on the complex nature of the disease and it can be caused by both genetic and environmental factors and with regard to genetic factors, HLA-Cw6 is the most important risk factor and provides the most significant relative risk. HLA associations were first reported in 1972 and since they have been consistently replicated. However, the distribution of HLA-Cw6 cannot explain the difference in prevalence among the various ethnic groups, since the highest population prevalence of this allele has been reported in blacks (15.09\%). The prevalence in Caucasoids is $9.62 \%$ and it is similar to Australian Aboriginals (9.05\%). Orientals (6.6\%) and Amerindians $(6.65 \%)$ are reported to have lower prevalence in similar level. Hence, HLA-Cw6 and psoriasis have a lower prevalence. Thus, other genetic factors and environmental factors may probably explain the observed differences and warrants further investigation of the disease.

\section{GWAS of psoriasis and its importance}

Genome-Wide Association Studies (GWAS) are currently the most popular in identifying susceptibility genes for complex diseases and Interleukin (IL) 12B on chromosome 5q and IL23R genes on chromosome $1 \mathrm{p}$ were identified using GWAS with a 25,215 genecentric SNP platform for discovery and a tag SNP for sequencing, this study confirmed a reported case of psoriasis-associated SNPs in the IL12B, i.e. rs 3212227 which is located at $30 \mathrm{~kb}$ untranslated region and rs6887695 which is located at $60 \mathrm{~kb}$ upstream. ${ }^{75}$ This study has also identified 2 missense SNP in IL23R and it is associated with psoriasis. In a Collaborative Association Study of Psoriasis, a cohort of 2845 subjects ( 1409 cases and 1436 controls) were analyzed using 438,670 autosomal SNPs and 2.5million imputed SNPs and these SNPs were tested for replication in a cohort consisting of 10,099 subjects among which 5048 are cases and 5051 contain healthy controls. ${ }^{75}$ Evidence of association was found at 10 of the 18 loci and compelled at the $7^{\text {th }}$ loci with a combined $\mathrm{P}$ value which is less than 0.05 . Initial comparison of case control allele frequencies confirmed association at following established susceptibility loci, i.e. HLA-C (rs12191877), IL12B (rs2082412) and IL23R (rs2201841) and other loci with confirmed association includes IL23A, TNAIP3 (TNF- $\alpha$ induced protein 3), TNIP1 (TNFAIP3 interacting protein 1), IL4 and IL13. Thus, in addition to HLA-C, three pathways of psoriasis susceptibility were identified i.e. TH17 pathway of IL12B (encoding the p40 subunit of IL23 \& IL12), IL23A (encoding the p19 subunit of IL23) and IL23R (encoding a subunit of the IL23 receptor) and NFkB pathway of TNFAIP3 and TNIP1 and TH2 pathway of IL4 and IL13). However, the association of signals identified in this study accounted the familial component of psoriasis and much familial components are required to be determined.

The vital role of genes in psoriasis has been recognized based on linkage studies and candidate gene studies. Recent advances in genetic analyses implicate the fact that, certain components are responsible for the regulation of IL-23 and NFKB pathways and those components may act as risk factors of psoriasis. Hence based on this study, researchers have advanced their understanding towards the nature of this complex disease. The above mentioned inflammatory pathways exhibit an increased activity in skin lesions and promote the secretion of various cytokines such as IL-17 and IL-22. Initially, the importance of genes in psoriasis had been recognized by traditional approaches of family-based linkage studies and population-based candidate gene association studies and it was thought that risk alleles of Psoriasis (HLA B*13, HLA B*17, B*37 HLA-B and HLA $\left.\mathrm{CW}^{*} 0602\right)$ are associated only with class I MHC molecule located on chromosome 6. Later SNP based Genome wide association approach is a great revolution in the identification of susceptible genes responsible for complex diseases like Psoriasis. According to SNP based genome wide approach, it has been found that, risk factors in non HLA genes (IL12B,IL23R,IL23A,IL13,TNFAIP3 and TNIP1) are also involved in causing Psoriasis. The list of Psoriasis susceptible Loci identified from Genome Wide Association Study is given in Table 1.

Table I Psoriasis susceptible Loci identified from Genome Wide Association Study

\begin{tabular}{|c|c|c|c|c|}
\hline SNP & Chromosomal location & Position (Mb) & Alleles risk/non-risk & Gene (Relative position) \\
\hline rs12191877 & $6 p 21.33$ & 31.36 & $\mathrm{~T} / \mathrm{C}$ & HLA-C (-I3kb) Intergenic \\
\hline rs20824I2 & $5 q 33.3$ & 158.65 & $\mathrm{G} / \mathrm{A}$ & ILI2B (+24kb) Intergenic \\
\hline rs220I84I & $|\mathrm{p} 3| .3$ & 67.47 & $\mathrm{G} / \mathrm{A}$ & IL23R Intronic \\
\hline rs2066808 & $12 q 13.3$ & 55.02 & $\mathrm{~A} / \mathrm{G}$ & IL23A (+3.7kb) Intronic \\
\hline rs2054l & $5 q 31.1$ & 132.02 & $\mathrm{G} / \mathrm{A}$ & ILI 3 Missense \\
\hline rs610604 & $6 q 23.3$ & 138.24 & $\mathrm{G} / \mathrm{T}$ & TNFAIP3 Intronic \\
\hline rs 17728338 & $5 q 33.1$ & 150.46 & $A / G$ & TNIPI (-I 2kb) Intergenic \\
\hline rs|2191877 & $6 \mathrm{p} 21.33$ & 31.36 & $\mathrm{~T} / \mathrm{C}$ & HLA-C (-I3kb) Intergenic \\
\hline rs20824I2 & $5 q 33.3$ & 158.65 & $\mathrm{G} / \mathrm{A}$ & ILI2B (+24kb) Intergenic \\
\hline rs220I84I & $|\mathrm{p} 3| .3$ & 67.47 & $\mathrm{G} / \mathrm{A}$ & IL23R Intronic \\
\hline rs2066808 & $12 q 13.3$ & 55.02 & $\mathrm{~A} / \mathrm{G}$ & IL23A (+3.7kb) Intronic \\
\hline rs2054l & $5 q 31.1$ & 132.02 & $\mathrm{G} / \mathrm{A}$ & ILI 3 Missense \\
\hline rs610604 & $6 q 23.3$ & 138.24 & $\mathrm{G} / \mathrm{T}$ & TNFAIP3 Intronic \\
\hline rs|7728338 & $5 q 33.1$ & 150.46 & $\mathrm{~A} / \mathrm{G}$ & TNIPI (-I2kb) Intergenic \\
\hline
\end{tabular}




\section{Conclusions and future perspectives of psoriasis}

US reports suggest that the prevalence of psoriasis is more common in women than men (Prevalence rate is less than $0.2 \%$ when compared with global prevalence i.e. $2 \%$ ) but according to Indian reports suggest that the prevalence is twice more common in men (Prevalence rate is less than $0.05 \%$ when compared with global prevalence i.e. $2 \%$ ) and the earlier onset of the disease is prior to 15 years.

In the era of Pre-genomics, studies done on Candidate gene of Psoriasis i.e. HLA-Cw6 was thought as the most important risk factor but the distribution of HLA-Cw6 cannot explain the difference in prevalence among the various ethnic groups. Hence, HLA-Cw6 and psoriasis have less prevalence and hence a genome wide association approach was applied for studying the prevalence of psoriasis in non HLA genes and it has been found that the additional genetic risk factors are IL12B,IL23R,IL23A,IL13,TNFAIP3 and TNIP1.

In the era of Post-genomics, genome wide association study had produced a lot of promising results for understanding the involvement of various risk factors responsible for causing complex auto-immune disorders like Psoriasis but much studies are not done to improve their level of significance towards the understanding of disease pathology.

Till date, most of the genomic studies have been underpowered to produce reliable results because they have not gone towards achievement of protocol development for "Personalized medicine". Future Perspectives of research in Psoriasis can focus on the development of predictive models for treating individual patients with Psoriasis.

\section{Acknowledgements}

None.

\section{Conflict of interest}

The author declares no conflict of interest.

\section{References}

1. Allen MH, Veal C, Faassen A, et al. A non-HLA gene within the MHC in psoriasis. Lancet. 1999;353(9164):1589-1590.

2. Ryan C, Bowcock A, Menter A. Use of pharmacogenomics in psoriasis Clin. Invest. 2011;1:399-411.

3. Doe PT, Asiedu A, Acheampong JW, et al. Skin diseases in Ghana and the UK. Int J Dermatol. 2001;40(5):323-326.

4. Eichelbaum M, Ingelman-Sundberg M, Evans WE. Pharmacogenomics and individualized drug therapy. Ann Rev Med. 2006;57:119-137.

5. Farber EM, Nall L. Psoriasis in the Tropics. Epidemiologic, genetic, clinical and therapeutical aspects. Dermatol Clin. 1994;12(4):805-816.

6. Gelfand JM, Stern RS, Nijsten T, et al. The prevalence of psoriasis in African Americans: Results from a population-based study. $J$ Am Acad Dermatol. 2005;52(1):23-26.

7. Griffiths CE, Barker JN. Pathogenesis and clinical features of psoriasis. Lancet. 2007;370(9583):263-271.

8. Parnami N, Garg T, Rath G, et al. Artificial cells, Development and characterization of nanocarriers for topical treatment of psoriasis by using combination therapy. Artificial cells Nanomedicine and Biotechnology. 2014;42:406-412.

9. Cevc G, Blume G. New, highly efficient formulation of diclofenac for the topical, transdermal administration in ultradeformable drug carriers, transfersomes. Biochim Biophys Acta. 2001;1514(2):191-205.
10. Cevc G, Blume G. Lipid vesicles penetrate into intact skin owing to the transdermal osmotic gradients and hydration force. Biochim Biophys Acta. 1992;1104(1):226-232.

11. Cevc G. Drug delivery across the skin. Exp Opin Investig Drugs. 1997;6(12):1887-1937.

12. Cevc G. Self-regulating 'smart carriers' for non-invasive and targeted drug delivery. CELL MOL BIOL Lett. 2002;7(2):224-225.

13. Touitou E, Dayan N, Bergelson L, et al. Ethosomes-novel vesicular carriers for enhanced delivery: characterization and skin penetration properties. J Control Release. 2000;65(3):403-418.

14. Touitou E, Godin B, Dayan N, et al. Intracellular delivery mediated by an ethosomal carrier. Biomaterials. 2001;22(22):3053-3059.

15. Morganti P, Ruocco E, Wolf R, et al. Percutaneous absorption and delivery systems. Clin Dermatol. 2001;19(4):489-501.

16. Hadgraft J. Recent developments in topical and transdermal delivery. Eur J Drug Metab Pharmacokinet. 1996;21(2):165-173.

17. Talegaonkar S, Azeem A, Ahmad FJ, et al. Microemulsions: a novel approach to enhanced drug delivery. Recent Pat Drug Deliv Formul. 2008;2(3):238-257.

18. Caubet C, Jonca N, Brattsand M, et al. Degradation of corneodesmosome proteins by two serine proteases of the kallikrein family, SCTE/KLK5/ hK5 and SCCE/KLK7/hK7. J Invest Dermatol. 2004;122 (5):1235-1244.

19. Elias PM, Friend DS. The permeability barrier in mammalian epidermis. J Cell Biol. 1975;65(1):180-191.

20. Prow TW, Chen X, Prow NA, et al. Nanopatch-targeted skin vaccination against West Nile Virus and Chikungunya virus in mice. Small. 2010;6(16):1776-1784

21. Monteiro-Riviere NA, Oldenburg SJ, Inman AO. Interactions of aluminum nanoparticles with human epidermal keratinocytes. $J$ Appl Toxicol. 2010;30(3):276-285.

22. Leavens TL, Xia XR, Lee HA, et al. Evaluation of perfused porcine skin as a model system to quantitate tissue distribution of fullerene nanoparticles. Toxicol Lett. 2010;197(1):1-6.

23. Naoui W, Bolzinger MA, Pelletier J, et al. Impact of microemulsion microstructure on drug absorption into skin. J Pharm Pharmacol. 2010;62(2010):788.

24. Fernando GJ, Chen X, Prow TW, et al. Potent immunity to low doses of influenza vaccine by probabilistic guided micro-targeted skin delivery in a mouse model. PLoS One. 2010;5(4):e10266.

25. Prow TW, Grice JE, Lin LL, et al. Nanoparticles and microparticles for skin drug delivery. Adv Drug Deliv Rev. 2011;63(6):470-491.

26. Esposito E, Menegatti E, Cortesi R. Ethosomes and liposomes as topical vehicles for azelaic acid: a preformulation study. J Cosmet Sci 2004;55(3):253-264

27. Yang F, Kamiya N, Goto M. Transdermal delivery of the anti-rheumatic agent methotrexate using a solid-in-oil nanocarrier. Eur J Pharm Biopharm. 2012;82(1):158-163.

28. Fernandez-Valverde SL, Taft RJ, Mattick JS. Dynamic isomiR regulation in Drosophila development. RNA. 2010;16(10):1881-1888.

29. Friedman RC, Farh KK, Burge CB, et al. Most mammalian mRNAs are conserved targets of microRNAs. Genome Res. 2009;19(1):92-105.

30. Fuchs E. Scratching the surface of skin development. Nature 2007;445(7130):834-842.

31. Slominski A, Zbytek B, Nikolakis G, et al. Steroidogenesis in the skin: Implications for local immune functions. J Steroid Biochem Mol Biol. 2013;137:107-123. 
32. Slominski AT, Zmijewski MA, Zbytek B, et al. Key role of CRF in the skin stress response system. Endocr Rev. 2013;34(6):827-884.

33. Berezikov E, Chung WJ, Willis J, et al. Mammalian mirtron genes. Mol Cell. 2007;28(2):328-336.

34. Bhalerao J, Bowcock AM. The genetics of psoriasis: a complex disorder of the skin and immune system. Hum Mol Genet. 1998;7(10):1537-1545.

35. Bissels U, Wild S, Tomiuk S, et al. Absolute quantification of microRNAs by using a universal reference. $R N A$. 2009;15(12):2375-2384.

36. Brameier M, Herwig A, Reinhardt R, et al. Human box C/D snoRNAs with miRNA like functions: expanding the range of regulatory RNAs. Nucleic Acids Res. 2011;39(2):675-686.

37. Gudjonsson JE, Ding J, Johnston A, et al. Assessment of the psoriatic transcriptome in a large sample: additional regulated genes and comparisons with in vitro models. J Invest Dermatol. 2010;130(2010):18291840 .

38. Miyoshi K, Miyoshi T, Siomi H. Many ways to generate microRNA-like small RNAs: non-canonical pathways for microRNA production. $\mathrm{Mol}$ Genet Genomics. 2010;284(2):95-103.

39. Wang H, Yang H, Shivalila CS, et al. One-step generation of mice carrying reporter and conditional alleles by crispr/cas-mediated genome engineering. Cell. 2013;154(4):1370-1379.

40. Hwu WL, Yang CF, Fann CS, et al. Mapping of psoriasis to $17 \mathrm{q}$ terminus. J Med Genet. 2005;42(2):152-158.

41. Zibert JR, Løvendorf MB, Litman T, et al. MicroRNAs and potential target interactions in psoriasis. J Dermatol Sci. 2010;58(3):177-185.

42. Bartel DP. MicroRNAs: target recognition and regulatory functions. Cell. 2009;136(2):215-233.

43. Carthew RW, Sontheimer EJ. Origins and Mechanisms of miRNAs and siRNAs. Cell. 2009;136(4):642-655.

44. Krek A, Grün D, Poy MN, et al. Combinatorial microRNA target predictions. Nat Genet. 2005;37(5):495-500.

45. Farh KK, Grimson A, Jan C, et al. The Widespread impact of Mammalian MicroRNAs on mRNA repression and evolution. Science. 2005;310(5755):1817-1821.

46. Czech MP. MicroRNAs as therapeutic targets. $N$ Engl J Med. 2006;354(11):1194-1195.

47. Gudjonsson JE, Ding J, Johnston A, et al. Assessment of the psoriatic transcriptome in a large sample: additional regulated genes and comparisons with in vitro models. $J$ Invest Dermatol. 2010;130(7):1829-1840.

48. Halsall JA, Osborne JE, Pringle JH, et al. Vitamin D receptor gene polymorphisms, particularly the novel A-1012G promoter polymorphism, are associated with vitamin D3 responsiveness and non-familial susceptibility in psoriasis. Pharmacogenet Genomics. 2005;15(5):349-355.

49. Huerta C, Rivero E, Rodríguez LA. Incidence and risk factors for psoriasis in the general population. Arch Dermatol Vol. 2007;143(12):15591565 .

50. Icen M, Crowson CS, McEvoy MT, et al. Trends in incidence of adultonset psoriasis over three decades: a population-based study. J Am Acad Dermatol. 2009;60(3):394-401.

51. Elder JT, Bruce AT, Gudjonsson JE, et al. Molecular dissection of psoriasis: integrating genetics and biology. $J$ Invest Dermatol. 2010;130(5):1213-1226.

52. Naldi L, Colombo P, Placchesi EB, et al. Study design and preliminary results from the pilot phase of the PraKtis study: self-reported diagnoses of selected skin diseases in a representative sample of the italian population. Dermatology. 2004;208(1):38-42.

53. Nall L, Gulliver W, Charmley P, et al. Search for the psoriasis susceptibility gene: the Newfoundland study. Cutis. 1999;64(5):323-329.
54. Nestle FO, Kaplan DH, Barker J. Psoriasis. $N$ Engl J Med. 2009;3615):496-509.

55. Nevitt GJ, Hutchinson PE. Psoriasis in the community: prevalence, severity and atients' beliefs and attitudes towards the disease. Br J Dermatol. 1996;135(4):533-537.

56. Ogunbiyi AO, Daramola OO, Alese OO. Prevalence of skin diseases in Ibadan, Nigeria. Int J Dermatol. 2004;43(1):31-36.

57. Olsen AO, Gribovski A, Magnus P, et al. Psoriasis in Norway as observed in a population-based Norwegian twin panel. Br J Dermatol. 2005;153(2):346-351.

58. Piskin G, Sylva-Steenland RM, Bos JD, et al. In Vitro and In Situ Expression of IL-23 by lesions: enhanced expression in psoriatic keratinocytes in healthy skin and psoriasis skin. J Immunol. 2005;176(3):1908-1915.

59. Pitchappan RM, Kakkaniah VN, Manickasundari M, et al. Susceptibility of major groups of Tamil Nadu to disease: Psoriasis vulgaris. Jounal of Genetics. 1989;68(2):75-92.

60. Plunkett A, Marks R. A review of the epidemiology of psoriasis vulgaris in the community. Australas J Dermatol. 1998;39(4):225-232.

61. Raychaudhuri SP. Farber EM The prevalence of psoriasis in the world. $J$ Eur Acad Dermatol Venereol. 2001;15(1):16-17.

62. Rhodri L1 Smith, Richard B Warren, Christopher EM Griffiths, Jane Worthington. Genetic susceptibility to psoriasis: an emerging picture. Genome Medicine. 2009;1(7):72.

63. Russell TJ, Schultes LM, Kuban DJ. Histocompatibility(HL-A): antigens associated with psoriasis. New England Jornal of Medicine. 1972;287:738-740.

64. Schäfer T. Epidemiology of psoriasis. Review and the German perspective. Dermatology. 2006;212(4):327-337.

65. Schön MP, Boehncke WH. Psoriasis. N Engl J Med. 2005;352(18):1899_ 1912.

66. Suite M. The Epidemiology of psoriasis in a dermatology clinic in a general hospital in port-of-Spain, Trinidad and Tobago, west Indies. West Indian Med J. 2006;55(6):399-402.

67. Tiilikainen A, Lassus A, Karvonen J, et al. Psoriasis and HLA-Cw6. Br J Dermatol. 1980;102(2):179-184.

68. Chandran V, Raychaudhuri SP. Geoepidemiology and environmental factors of psoriasis and psoriatic arthritis. J Autoimmun. 2010;34(3):J314 $\mathrm{J} 321$.

69. Vinod Chandran. Genetics of psoriasis and psoriatic arthritis. Indian $J$ Dermatol. 2100;55(2):151-156.

70. Warren RB, Smith RL, Campalani E, et al. Genetic variation in efflux transporters influences outcome to methotrexate therapy in patients with psoriasis. J Invest Dermatol. 2008;128(8):1925-1929.

71. Young HS, Summers AM, Read IR, et al. Interaction between genetic control of vascular endothelial growth factor production and retinoid responsiveness in psoriasis. J Invest Dermatol. 2006;126(2):453-459.

72. Creamer D, Allen M, Jaggar R, et al. Mediation of systemic vascular hyperpermeability in severe psoriasis by circulating vascular endothelial growth factor. Arch Dermatol. 2002;138(6):791-796.

73. Creamer D, Allen MH, Groves RW, et al. Circulating vascular permeability factor/vascular endothelial growth factor in erythroderma. Lancet. 1996;348(9034):1101.

74. Creamer D, Allen MH, Sousa A, et al. Localization of endothelial proliferation and microvascular expansion in active plaque psoriasis. $\mathrm{Br} J$ Dermatol. 1997;136(6):859-865.

75. Creamer JD, Barker JNWN. Vascular proliferation and angiogenic factors in psoriasis. Clin Exp Dermatol. 1995;20:6-9. 